\title{
Sarcopenia as a predictor of major hepatectomy complications: a little addition
}

\author{
Umit Cintosun $^{1} \cdot$ Battal Altun $^{2}$
}

Received: 5 November 2015 / Accepted: 9 November 2015 / Published online: 26 November 2015

(C) Japan Society of Clinical Oncology 2015

To the Editor,

Higashi et al. [1] once again enlightened the importance of sarcopenia in oncological surgery patients. As the authors mentioned in the study, sarcopenia and visceral fat amount can be predictors for postoperative complications including mortality and morbidities like liver dysfunction. However, assessment of sarcopenia needs to be straightforward in such studies. The authors assessed sarcopenia by computerized tomography (CT) (L3 muscle index) based on the previous reports that this surrogate indicator yields successful estimation of whole-body skeletal muscle mass. Although muscle mass estimation with CT seems to be a valid approach, diagnosis of sarcopenia needs further consideration. Because, impaired muscle functions and strength which have gained significant importance in recent years are important components of sarcopenia. This was carefully highlighted by a consensus report from the European Working Group on Sarcopenia in Older People $[2,3]$. Along with the muscle mass, the group addressed physical performance as assessed by gait speed, and grip strength measured by handheld dynamometer to be used in the decision making for sarcopenia. Thus, using only L3 muscle index would cause false distinction between presarcopenia and sarcopenia. Secondly, the fixed wide ranges of attenuation values for defining muscle mass area in the study by Higashi et al. remain relatively broad that could result in false distinction of tissues [4]. Clarification of these issues may be relevant to the study results.

\section{Compliance with ethical standards}

Conflict of interest The authors declare that they have no conflict of interest.

\section{References}

1. Higashi T, Hayashi H, Taki K et al (2015) Sarcopenia, but not visceral fat amount, is a risk factor of postoperative complications after major hepatectomy. Int J Clin Oncol. doi:10.1007/ s10147-015-0898-0

2. Cruz-Jentoft AJ, Baeyens JP, Bauer JM et al (2010) Sarcopenia: European consensus on definition and diagnosis: report of the European Working Group on Sarcopenia in Older People. Age Ageing 39:412-423

3. Cintosun U, Tasci I, Binay Safer V et al (2015) Sarcopenia is more than simply documenting low skeletal muscle mass. Cancer 121(1): 159

4. Parkin E, Plumb AA, O'Reilly D et al (2012) Body composition and outcome in patients undergoing resection of colorectal liver metastases. Br J Surg 99:550-557
Umit Cintosun

drumitcintosun@gmail.com

1 Department of Geriatrics, Gulhane School of Medicine, GATA Geriatri B.D., 06018 Etlik, Ankara, Turkey

2 Department of Internal Medicine, Kasımpasa Military Hospital, 34440 Istanbul, Turkey 